\title{
Clinical use of sodium-glucose co-transporter-2 inhibitors in Chinese patients with type 2 diabetes mellitus
}

Wing Bun Chan, MBChB, FRCPG

\begin{abstract}
INTRODUCTION Type 2 diabetes mellitus (T2DM), the tenth leading cause of death in Hong Kong, has a prevalence of approximately $10 \%$. Sodium-glucose co-transporter-2 (SGLT2) inhibitors lower glycated haemoglobin (HbA1c) levels in T2DM patients via a non-insulin-dependent mechanism of action, but real-world data is limited, particularly for Chinese patients.

METHODS A retrospective single-centre study was performed among Chinese patients with T2DM who were prescribed SGLT2 inhibitor therapy in Hong Kong. Changes in HbA1c levels, body weight, systolic and diastolic blood pressure, estimated glomerular filtration rate (eGFR), lipid profiles and adverse events were observed for patients who completed at least one follow-up visit during the study period.

RESULTS Overall, 100 patients were included, and 53 patients attended an additional final visit. By the final visit, SGLT2 inhibitor therapy had significantly decreased $\mathrm{HbA} 1 \mathrm{c}$ levels (change $[\Delta] 0.31 \%, 95 \%$ confidence interval $[\mathrm{Cl}]-0.11 \%$ to $-0.51 \%, p<0.001)$, body weight $(\Delta-4.59 \mathrm{~kg}, 95 \% \mathrm{Cl}-3.75$ to $-5.54 \mathrm{~kg}, \mathrm{p}<0.001)$ and systolic blood pressure $(\Delta-5.72 \mathrm{mmHg}, 95 \% \mathrm{Cl}-1.72$ to $-9.72 \mathrm{mmHg}, \mathrm{p}<0.001)$ from baseline. No significant change in eGFR or lipid profiles was observed, except for a significant reduction in high-density lipoprotein cholesterol $(\Delta-0.09 \mathrm{mmol} / \mathrm{L}, 95 \% \mathrm{Cl}-0.16$ to $-0.02 \mathrm{mmol} / \mathrm{L}, \mathrm{p}<0.05)$. Adverse events were consistent with previous reports for SGLT2 inhibitors, apart from appetite loss associated with canagliflozin.

CONCLUSION The real-world efficacy and safety profile of SGLT2 inhibitors in Chinese patients was comparable to that reported in Phase III clinical trials, with the exception of appetite loss among patients who received canagliflozin.
\end{abstract}

Keywords: blood pressure, body weight, glycated haemoglobin, hyperglycaemia, type 2 diabetes mellitus

\section{INTRODUCTION}

Diabetes mellitus is a global epidemic affecting 422 million people worldwide, or approximately $8.5 \%$ of the global population, ${ }^{(1)}$ and is associated with an increased risk of cardiovascular disease, kidney failure, retinopathy and neuropathy. In 2012 alone, diabetes mellitus was designated as the cause of death for 1.5 million people worldwide, while elevated blood glucose was responsible for an additional 2.2 million deaths. ${ }^{(1)}$ These statistics are also reflected in Hong Kong, where over 580,000 people aged $20-79$ years, or approximately $10 \%$ of its adult population, have type 2 diabetes mellitus (T2DM), which is listed as the tenth leading cause of death. ${ }^{(2,3)}$

T2DM is characterised by hyperglycaemia resulting from resistance to insulin - the hormone that stimulates glucose uptake in the liver, muscles and adipose tissue - and/or a relative insulin deficiency, especially in patients with obesity. ${ }^{(4)}$ The degree of hyperglycaemia in patients with T2DM has, in turn, been correlated with several cardiovascular and clinical outcomes. ${ }^{(5)}$

Therefore, the primary aim of treatment for patients with T2DM is to maintain glycaemic control with lifestyle modification and pharmacological interventions. While metformin is considered to be the first-line treatment for the pharmacological treatment of T2DM, the relationship between insulin-dependent pharmacological interventions and disease progression in T2DM is complex. ${ }^{(4)}$ Oral antidiabetic drugs (OADs) with an insulindependent mechanism of action, such as sulphonylureas (SUs) and incretin mimetics (dipeptidyl peptidase-4 [DPP4] inhibitors and glucagon-like peptide- 1 agonists), do not address the underlying insulin resistance, and incretin mimetics are also associated with gastrointestinal adverse events, such as diarrhoea and nausea and, in rare cases, pancreatitis. ${ }^{(4)}$ Furthermore, SUs, thiazolidinediones (TZDs) and exogenous insulin are also associated with weight gain. Given that SUs also stimulate the release of insulin independently of the presence of glucose, both SUs and exogenous insulin are also associated with an increased risk of hypoglycaemia, which, in turn, has been linked to an increased risk of major cardiovascular events among patients with T2DM. ${ }^{(4,6)}$

In contrast, sodium-glucose co-transporter-2 (SGLT2) inhibitors are a new class of OADs offering a non-insulindependent mechanism of action by inhibiting the reabsorption of glucose in the glomerular filtrate in the kidneys, allowing excess glucose to be excreted in the urine. ${ }^{(7)}$ This class of drugs has been shown to be efficacious in reducing glycated haemoglobin (HbA1c) levels in patients with T2DM, while also offering the additional benefit of weight loss, blood pressure reduction and increased high-density lipoprotein cholesterol levels, independent of a patient's degree of $\beta$-cell function or insulin sensitivity. ${ }^{(8)}$ Furthermore, recent data not only suggests that the efficacy of SGLT2 inhibitors observed during Phase III clinical trials is replicated in a real-world setting, but also that this class of drugs may be more efficacious than DPP4 inhibitors. ${ }^{(9,10)}$

While SGLT2 inhibitors require sufficient renal function to be efficacious, no adverse impact on renal function has been reported 
within this drug class. In fact, it has been suggested that SGLT2 inhibitors may have a nephroprotective effect by reducing serum uric acid levels, tubular glucose toxicity and diabetes mellitusrelated hyperfiltration, as evidenced by reduced albuminuria in patients who were administered SGLT2 inhibitors in combination with renin-angiotensin system blockers. ${ }^{(11)}$ Likewise, the first prospective cardiovascular outcomes study for a SGLT2 inhibitor, the EMPA-REG OUTCOME study of empagliflozin, reported a significant reduction in the risk of major cardiovascular events compared with a placebo, although it is not yet clear whether this is a class effect, as prospective cardiovascular outcome studies for dapagliflozin and canagliflozin are ongoing. ${ }^{(12)}$ Furthermore, SGLT2 inhibitors are associated with a low risk of hypoglycaemia, and the most commonly observed adverse events, such as urinary tract infections (UTIs) and genital infections, are generally considered to be mild to moderate in severity and respond to standard treatment. ${ }^{(13)}$

However, real-world patterns of usage after a drug receives marketing approval are often different from those reported in clinical trials and are shaped by local policy and differing population characteristics, such as polypharmacy and comorbidities, when compared with the defined inclusion/ exclusion criteria used in clinical trials, as was observed in the Association of British Clinical Diabetologists nationwide exenatide audit, which found that approximately $40 \%$ of exenatide prescriptions were issued off-label, as an add-on to insulin therapy. ${ }^{(14)}$ Therefore, the current study aimed to assess the efficacy and safety of SGLT2 inhibitor therapy in a real-world population of patients of Chinese ethnicity with T2DM.

\section{METHODS}

The current study was a single-centre retrospective observational study of 100 patients with T2DM enrolled at a private specialist diabetes clinic comprising four physicians in the Special Administrative Region of Hong Kong, which was conducted as part of a regular clinical audit. Patients enrolled in the study were required to be of Chinese ethnicity, to be taking prescribed SGLT2 inhibitor therapy during an initial enrolment visit that occurred between 1 February 2015 and 30 November 2015, and to have attended at least one follow-up visit during this period. The SGLT2 inhibitor administered (canagliflozin, dapagliflozin or empagliflozin) was chosen at the physician's discretion. Most patients were followed up every 8-12 weeks at each individual physician's discretion as part of routine clinical practice, as there was no prespecified standardised follow-up schedule. The visit closest to and within three months was designated as the first follow-up visit and the last available visit after three months was designated as the final visit. As this was a retrospective observational study conducted as part of a clinical audit, written informed consent was not obtained.

As part of the routine clinical management of patients with T2DM, body weight and blood pressure were assessed at every visit, while HbA1c was monitored every 8-12 weeks. Lipid profiles were assessed every six months. Renal function tests were scheduled annually, with optional renal function tests performed at the physician's discretion after initiating SGLT2 inhibitor therapy. The methodology for estimating glomerular filtration rates was also chosen at the physician or laboratory's discretion.

Patients were routinely screened for known adverse events associated with the SGLT2 class of OADs, including UTIs, vaginitis, balanitis and postural hypotension. Individual patient data relating to clinical history, concomitant drug utilisation, anthropometric parameters, clinical parameters, biochemical parameters and adverse events were extracted from the case notes of patients.

Data is presented as mean \pm standard deviation. Analysis of variance and post-hoc paired $t$-tests were performed to compare treatment efficacy. Regression analysis was used to explore relationships between baseline characteristics and treatment efficacy. All statistical analyses were performed using SPSS version 14 (SPSS Inc, Chicago, IL, USA). All analyses were exploratory.

\section{RESULTS}

The demographic and baseline clinical characteristics of the 100 patients included in this study are presented in Table I. Among these, 46 patients were administered SGLT2 therapy as an add-on to their previous antidiabetic medication, while 54 patients were prescribed an SGLT2 inhibitor to replace previously prescribed therapy. Patients administered SGLT2 inhibitor therapy as an add-on were observed to have significantly higher $\mathrm{HbA} 1 \mathrm{c}$ levels $(p=0.005)$ and systolic blood pressure $(p=0.025)$ compared with those who were prescribed SGLT2 inhibitor replacement therapy.

As might be expected, patients who were administered SGLT2 inhibitor add-on therapy were also prescribed a significantly greater number of concomitant medications $(p=0.019)$. A majority of patients were prescribed SGLT2 inhibitor to improve glycaemic control $(76.0 \%)$; however, for $25.9 \%(n=14)$ of patients from the SGLT2 inhibitor replacement therapy group, this was because of adverse events after previous medication (Table II), including oedema (TZDs, $n=9$ ), hypoglycaemia ( $S U$, $\mathrm{n}=3$ ), epigastric discomfort (metformin, $\mathrm{n}=1$ ) and skin reaction (DPP4 inhibitor, $\mathrm{n}=1$ ).

Patients were followed up for a mean duration of 22.7 weeks following the index visit, with a mean duration of 9.9 weeks between the index and first follow-up visits. Data was also available for 53 patients who attended a final visit following the first follow-up visit (add-on therapy, $\mathrm{n}=28$, replacement therapy, $\mathrm{n}=25$ ).

SGLT2 inhibitor therapy significantly decreased HbA1c levels by $0.31 \%$ (95\% confidence interval $[\mathrm{Cl}]-0.11 \%$ to $0.51 \%$, $\mathrm{p}<0.001$ ) between the baseline and final visits (Fig. 1a). A similar effect was also observed in patients who were administered SGLT2 inhibitor add-on therapy (change $[\Delta]-0.63 \%, 95 \% \mathrm{Cl}-0.35 \%$ to $0.91 \%, \mathrm{p}<0.001$ ), whereas a transient reduction in $\mathrm{HbA} 1 \mathrm{c}$ levels was observed at the first follow-up visit in patients receiving replacement therapy $(\Delta-0.28 \%, 95 \% \mathrm{Cl}-0.08 \%$ to $-0.48 \%$, $\mathrm{p}<0.01$ ) that was not maintained at the final visit $(\Delta-0.29 \%$, $95 \% \mathrm{Cl}-0.02 \%$ to $-0.54 \%, \mathrm{p}=0.18$ ). In particular, no significant changes in $\mathrm{HbA} 1 \mathrm{c}$ levels were observed among patients in whom an SGLT2 inhibitor replaced previous SU $(\Delta+0.13 \%)$, 
Table I. Patient demographics and clinical characteristics at baseline.

\begin{tabular}{|c|c|c|c|c|}
\hline \multirow[t]{2}{*}{ Variable } & \multicolumn{3}{|c|}{ No. (\%)/mean \pm SD (range) } & \multirow[t]{2}{*}{ p-value } \\
\hline & Overall $(n=100)$ & $\begin{array}{l}\text { SGLT2 add-on } \\
\text { therapy }(n=46)\end{array}$ & $\begin{array}{l}\text { SGLT2 replacement } \\
\text { therapy }(n=54)\end{array}$ & \\
\hline Age (yr) & $57.4 \pm 12.1(29-85)$ & $56.7 \pm 11.6(29-82)$ & $58.0 \pm 12.5(30-85)$ & 0.604 \\
\hline Male gender & $57(57.0)$ & $25(54.3)$ & $32(59.3)$ & 0.621 \\
\hline Time since diagnosis* (yr) & $15.25 \pm 7.84$ & $15.46 \pm 8.12$ & $15.06 \pm 15.06$ & \\
\hline HbA1c (\%) & $7.85 \pm 0.92(5.4-10.1)$ & $8.12 \pm 0.95(6.3-10.1)$ & $7.61 \pm 0.85(5.4-9.8)$ & $0.005^{+}$ \\
\hline BMI $\left(\mathbf{k g} / \mathbf{m}^{2}\right)$ & $28.3 \pm 4.9(16.5-46.9)$ & $29.0 \pm 4.8(18.3-39.3)$ & $28.3 \pm 4.9(16.5-46.9)$ & 0.231 \\
\hline \multicolumn{5}{|l|}{ Blood pressure (mmHg) } \\
\hline Systolic & $131 \pm 14(100-167)$ & $135 \pm 15(100-167)$ & $128 \pm 13(103-154)$ & $0.025^{\dagger}$ \\
\hline Diastolic & $80 \pm 12(45-110)$ & $82 \pm 12(45-100)$ & $78 \pm 12(52-110)$ & 0.084 \\
\hline eGFR $\left(\mathrm{mL} / \mathrm{min} / \mathrm{m}^{2}\right)$ & $94 \pm 26(45-165)$ & $98 \pm 27(45-165)$ & $90 \pm 26(45-164)$ & 0.161 \\
\hline Total cholesterol (mmol/L) & $3.83 \pm 0.83(2.50-7.38)$ & $3.75 \pm 0.86(2.50-6.60)$ & $3.89 \pm 0.81(2.50-7.38)$ & 0.404 \\
\hline Triglycerides (mmol/L) & $1.35 \pm 0.96(0.29-5.60)$ & $1.35 \pm 0.82(0.44-4.35)$ & $1.35 \pm 1.07(0.29-5.60)$ & 0.997 \\
\hline LDL-C (mmol/L) & $1.79 \pm 0.67(0.20-4.40)$ & $1.79 \pm 0.81(0.20-4.40)$ & $1.80 \pm 1.54(0.70-3.60)$ & 0.914 \\
\hline HDL-C (mmol/L) & $1.43 \pm 0.40(0.84-2.47)$ & $1.38 \pm 0.38(0.84-2.30)$ & $1.47 \pm 0.41(0.94-2.47)$ & 0.328 \\
\hline \multicolumn{5}{|l|}{ Comorbidity } \\
\hline Cardiovascular disease/CHD & $25(25.0)$ & $15(32.6)$ & $10(18.5)$ & 0.100 \\
\hline Retinopathy & $24(24.0)$ & $12(26.1)$ & $12(22.2)$ & 0.652 \\
\hline Albuminuria & $30(30.0)$ & $14(30.4)$ & $16(29.6)$ & 0.930 \\
\hline $\begin{array}{l}\text { Concomitant medications } \\
\text { prescribed }^{\ddagger}\end{array}$ & $2.65(0-4)$ & $2.82(1-4)$ & $2.50(0-4)$ & $0.019^{\dagger}$ \\
\hline Antihypertensive drug & $67(67.0)$ & $34(73.9)$ & $33(61.1)$ & 0.174 \\
\hline Lipid-lowering drug & $84(84.0)$ & $38(82.6)$ & $46(85.2)$ & 0.726 \\
\hline Injectable therapy prescribed & $51(51.0)$ & $24(52.8)$ & $27(50.0)$ & 0.960 \\
\hline \multicolumn{5}{|l|}{$\begin{array}{l}\text { Injectable antidiabetic therapy } \\
\text { prescribed }\end{array}$} \\
\hline GLP-1 receptor agonist alone & $1(1.0)$ & $0(0.0)$ & $1(1.9)$ & - \\
\hline Basal insulin alone & $42(42.0)$ & $24(52.2)$ & $18(33.3)$ & - \\
\hline Both GLP-1 and insulin & $8(8.0)$ & $2(4.3)$ & $6(11.1)$ & - \\
\hline \multicolumn{5}{|c|}{ SGLT2 inhibitor therapy prescribed } \\
\hline Canagliflozin & $45(45.0)$ & $23(50.0)$ & $22(40.7)$ & - \\
\hline Dapagliflozin & $41(41.0)$ & $18(39.1)$ & $23(42.6)$ & - \\
\hline Empagliflozin & $14(14.0)$ & $5(10.9)$ & $9(16.7)$ & - \\
\hline
\end{tabular}

*Data presented as mean \pm SD. $+p<0.05$ is statistically significant. $\neq$ Data presented as mean no. (range). BMI: body mass index; CHD: coronary heart disease; eGFR: estimated glomerular filtration rate; GLP-1: glucagon-like protein; HbA1c: glycated haemoglobin; HDL-C: high-density lipoprotein cholesterol; LDL-C: lowdensity lipoprotein cholesterol; SD: standard deviation; SGLT2: sodium-glucose co-transporter 2

TZD $(\Delta+0.22 \%)$ or DPP4 inhibitor therapy $(\Delta+0.05 \%)$, but a significant reduction was observed for patients for whom SGLT2 inhibitor replaced $\alpha$-glucosidase inhibitor therapy $(\Delta-0.69 \%$, $95 \% \mathrm{Cl}-0.45 \%$ to $-0.93 \%$, $\mathrm{p}<0.01$ ).

No significant difference in the changes in HbA1c levels was observed at the final visit between patients who were administered oral agents alone and those who were administered injectables, or in patients who were administered 1-2 compared to $\geq 3$ antidiabetic agents. Likewise, no significant difference was observed between the efficacy of empagliflozin, dapagliflozin or canagliflozin. Regression analyses did not identify any relationship between SGLT2 inhibitor therapy efficacy and body mass index (BMI) or estimated glomerular filtration rate (eGFR).

Significant weight loss was reported at the final visit after initiating SGLT2 inhibitor therapy $(\Delta-4.59 \mathrm{~kg}, 95 \% \mathrm{Cl}-3.75$ to
5.54 kg, p < 0.001 vs. baseline; Fig. 1b), which was consistently observed across patients who were administered add-on therapy $(\Delta-5.12 \mathrm{~kg}, 95 \% \mathrm{Cl}-3.67$ to $-6.57 \mathrm{~kg}, \mathrm{p}<0.05)$ and replacement therapy $(\Delta-4.58 \mathrm{~kg}, 95 \% \mathrm{Cl}-2.58$ to $-6.58 \mathrm{~kg}, \mathrm{p}<0.001)$. Systolic blood pressure was also reduced over the course of the study $(\Delta-5.72 \mathrm{mmHg}, 95 \% \mathrm{Cl}-1.72$ to $9.72 \mathrm{mmHg}, \mathrm{p}<0.001$ vs. baseline; Fig. 1c). The observed reduction in diastolic blood pressure did not reach significance $(\Delta-2.16 \mathrm{mmHg}, \mathrm{p}=0.146$ vs. baseline; Fig. 1c).

No significant changes in eGFR or lipid profiles were observed, except for a significant reduction in high-density lipoprotein cholesterol $(\Delta-0.09 \mathrm{mmol} / \mathrm{L}, 95 \% \mathrm{Cl}-0.02$ to $-0.16 \mathrm{mmol} / \mathrm{L}, \mathrm{p}<0.05$; Table III).

Adverse events reported by patients who were administered SGLT2 inhibitors were consistent with those previously reported. 
Table II. Reasons for prescribing SGLT2 inhibitor therapy.

\begin{tabular}{|c|c|c|}
\hline \multirow[t]{2}{*}{ Reason } & \multicolumn{2}{|c|}{ No. of patients } \\
\hline & $\begin{array}{l}\text { Add-on } \\
\text { therapy } \\
(n=46)\end{array}$ & $\begin{array}{l}\text { Replacement } \\
\text { therapy } \\
(n=54)\end{array}$ \\
\hline $\begin{array}{l}\text { Inadequate glycaemic } \\
\text { control }\end{array}$ & 40 & 36 \\
\hline Weight loss & 4 & 3 \\
\hline Adverse event & 0 & 14 \\
\hline To replace injectable therapy & 2 & 1 \\
\hline
\end{tabular}

SGLT2: sodium-glucose co-transporter 2

Table III. Key findings on renal function and lipid profiles at the final follow-up visit.

\begin{tabular}{|llll|}
\hline Variable & $\begin{array}{l}\text { No. of } \\
\text { patients }\end{array}$ & $\begin{array}{l}\text { Change from } \\
\text { baseline } \\
\text { (mean } \pm \text { SD) }\end{array}$ & p-value \\
\hline eGFR (mL/min/m ${ }^{2}$ ) & & & \\
\hline At first follow-up & 29 & $-3.65 \pm 15.4$ & 0.230 \\
\hline At final follow-up & 22 & $-5.32 \pm 17.5$ & 0.170 \\
\hline $\begin{array}{l}\text { Total cholesterol } \\
\text { (mmol/L) }\end{array}$ & 50 & $0.02 \pm 0.63$ & 0.819 \\
\hline Triglycerides (mmol/L) & 50 & $0.00 \pm 0.81$ & 0.993 \\
\hline LDL-C (mmol/L) & 50 & $0.14 \pm 0.56$ & 0.079 \\
\hline HDL-C (mmol/L) & 50 & $-0.09 \pm 0.26$ & $0.014^{*}$ \\
\hline
\end{tabular}

${ }^{*} \mathrm{p}<0.05$ is statistically significant. eGFR: estimated glomerular filtration rate; HDL-C: high-density lipoprotein cholesterol; LDL-C: Iow-density lipoprotein cholesterol

At the first visit, five patients had developed UTI and 16 patients reported vaginitis or balanitis. Of the five patients experiencing poor appetite, all were administered canagliflozin. At the final visit, no UTIs were reported, while six patients had vaginitis or balanitis. As at the first follow-up visit, the two patients experiencing poor appetite were both administered canagliflozin.

Seven patients discontinued SGLT2 inhibitor treatment after the first visit, while three additional patients had discontinued therapy at the final visit. Four patients discontinued treatment due to vaginitis or balanitis, while other patients discontinued treatment due to poor appetite and excessive weight loss $(n=3)$, malaise $(n=1)$, lack of efficacy $(n=1)$ and no longer needing treatment because of bariatric surgery $(n=1)$.

\section{DISCUSSION}

The current study presented real-world data of Chinese patients with T2DM who were treated with SGLT2 inhibitors, indicating a comparable efficacy and safety profile when compared with that reported in Phase III clinical trials. In particular, this real-world cohort included a high number of patients with advanced or complicated disease that was associated with diabetes mellitusrelated comorbidities and concomitant medications, and required treatment with multiple antidiabetic agents, including injectables. Notably, this study included patients who had been prescribed one of the three SGLT2 inhibitor therapies approved in Hong Kong at the time of this study: canagliflozin, dapagliflozin and
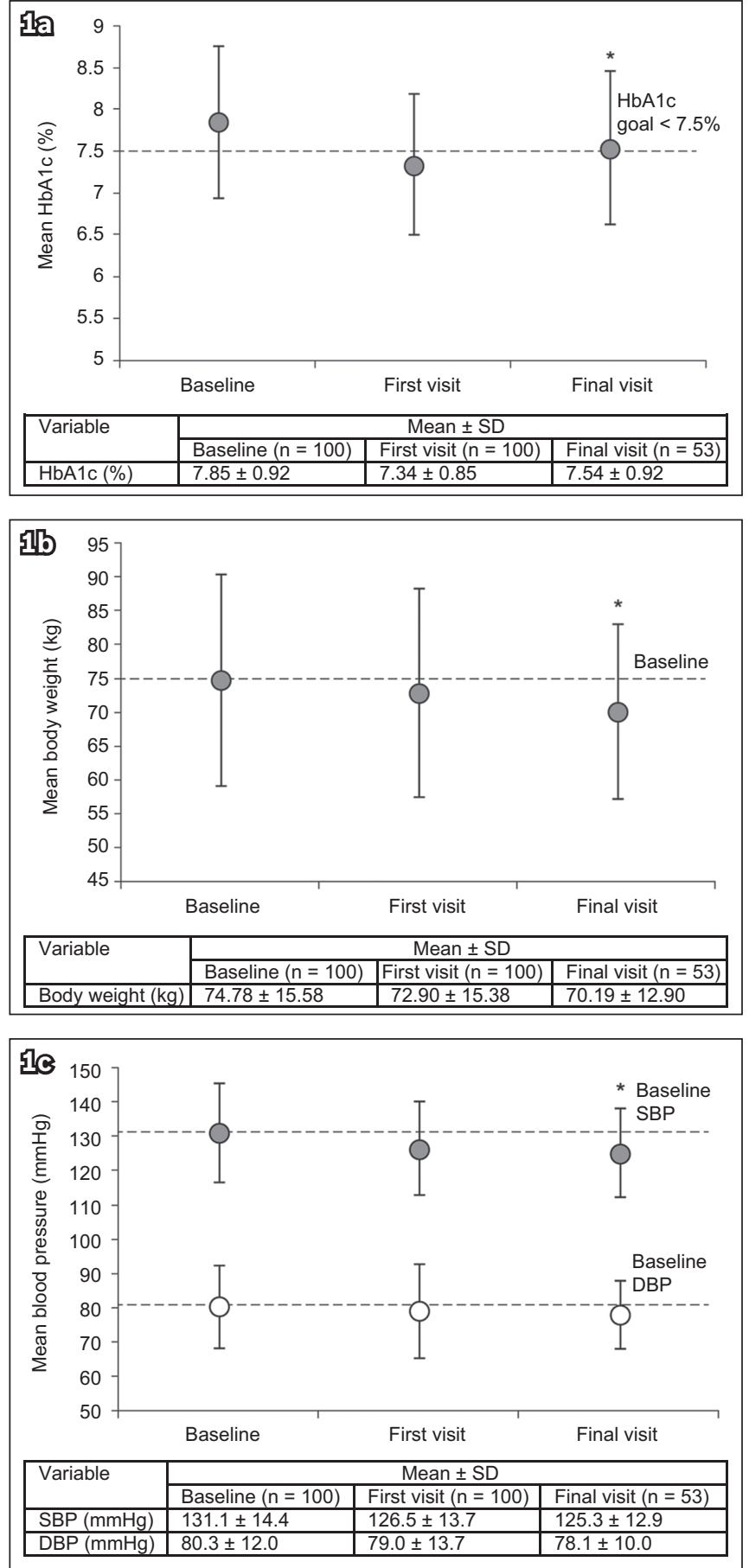

Fig. 1 Charts show overall changes from baseline to final follow-up visit in (a) HbA1c; (b) body weight; and (c) SBP and DBP levels. ${ }^{*} \mathrm{p}<0.001$ was statistically significant. DBP: diastolic blood pressure; HbA1c: glycated haemoglobin; SBP: systolic blood pressure; SD: standard deviation

empagliflozin. Prior to the study, to our knowledge, published data relating to the use of SGLT2 inhibitors to treat Chinese patients with T2DM was limited to a small study of dapagliflozin monotherapy in drug-naive patients. ${ }^{(15)}$

The efficacy of SGLT2 inhibitors appeared to be greater when they were prescribed as an add-on therapy rather than replacement therapy, although this was not unexpected given that the efficacy of antidiabetic therapies in improving glycaemic control is known to be dependent on baseline HbA1c levels. ${ }^{(16)}$ Furthermore, clinical trials have generally demonstrated the use 
of SGLT2 inhibitors as an add-on therapy in patients requiring improved glycaemic control and not as a replacement therapy for those who have adequate glycaemic control but are intolerant to their existing regimen. However, SGLT2 inhibitors offered the additional benefits of weight loss and lower blood pressure as both add-on and replacement therapies.

In contrast to a meta-analysis of clinical trials investigating SGLT2 inhibitor therapy in patients with T2DM, efficacy in the current study was not correlated with patient BMI and a significant decrease in high-density lipoprotein cholesterol levels was observed, as opposed to the previously reported increase. ${ }^{(8)}$ In addition, no correlation was found between efficacy and eGFR, suggesting that SGLT2 inhibitor therapy may be suitable for a broader range of patients than is defined by the current eGFRdependent approved indications.

However, as was expected from earlier reports, SGLT2 inhibitor therapy was associated with an increased prevalence of UTIs and genital infections in our study, although all UTIs had cleared between the first and final follow-up visits. Interestingly, loss of appetite reported by some patients who were administered canagliflozin does not appear to have been reported previously. As canagliflozin, but not dapagliflozin or empagliflozin, also inhibits sodium-glucose co-transporter-1 (SGLT1), ${ }^{(17)}$ which is expressed in the intestines, SGLT1 inhibition offers a hypothetical mechanism for this adverse event, and its initial reporting in our Chinese population warrants further investigation.

This study had several limitations due to its retrospective analysis of data, which was derived from a small sample at a single specialist centre where all patients were being treated by one of four physicians. This raises the risk of bias in the results reported, given the exploratory nature of all analyses performed. The applicability of this data in a primary care setting may also be limited. Furthermore, the follow-up interval and pattern of assessment were at the treating physician's discretion, so data from the final follow-up visit was not available for all patients, particularly with regard to some laboratory assessments, such as renal function tests.

In conclusion, the efficacy and safety profile of SGLT2 inhibitors prescribed to Chinese patients with T2DM, observed in the real world, was comparable to that reported in Phase III clinical trials, with the exception of the newly reported adverse event of appetite loss among patients administered canagliflozin. The current study also supports the use of SGLT2 inhibitors to treat a broad range of patients with T2DM encountered in clinical practice, including those with advanced or complicated disease.

\section{ACKNOWLEDGEMENTS}

This review was funded by AstraZeneca Hong Kong Limited, Hong Kong. Medical writing support was provided by Blair Hesp, PhD, CMPP, of MIMS (Hong Kong) Limited and funded by AstraZeneca.

\section{REFERENCES}

1. World Health Organization. Global report on diabetes. Available at: http:// www.who.int/diabetes/global-report/en/. Accessed June 5, 2019.

2. Healthy HK, Department of Health, Hong Kong. Diabetes mellitus. Available at: http://www.healthyhk.gov.hk/phisweb/en/healthy_facts/disease_burden/ major_causes_death/diabetes_mellitus/. Accessed June 5, 2019.

3. International Diabetes Federation. IDF Diabetes Atlas 7th edition (2015). Available at: https://www.idf.org/e-library/epidemiology-research/diabetesatlas/13-diabetes-atlas-seventh-edition.html. Accessed June 5, 2019.

4. Inzucchi SE, Bergenstal RM, Buse JB, et al; American Diabetes Association (ADA); European Association for the Study of Diabetes (EASD). Management of hyperglycemia in type 2 diabetes: a patient-centred approach: position statement of the American Diabetes Association (ADA) and the European Association for the Study of Diabetes (EASD). Diabetes Care 2012; 35:1364-79.

5. Stratton IM, Adler AI, Neil HA, et al. Association of glycaemia with macrovascular and microvascular complications of type 2 diabetes (UKPDS 35): prospective observational study. BMJ 2000; 321:405-12.

6. Zoungas S, Patel A, Chalmers J, et al; ADVANCE Collaborative Group. Severe hypoglycaemia and risks of vascular events and death. N Engl J Med 2010; 363:1410-8.

7. Inzucchi SE, Bergenstal RM, Buse JB, et al. Management of hyperglycemia in type 2 diabetes, 2015: a patient-centred approach: update to a position statement of the American Diabetes Association and the European Association for the Study of Diabetes. Diabetes Care 2015; 38:140-9.

8. Monami M, Nardini C, Mannucci E. Efficacy and safety of sodium glucose cotransport-2 inhibitors in type 2 diabetes: a meta-analysis of randomized clinical trials. Diabetes Obes Metab 2014; 16:457-66.

9. Wilding J, Bailey C, Rigney U, et al. Glycated hemoglobin, body weight and blood pressure in type 2 diabetes patients initiating dapagliflozin treatment in primary care: a retrospective study. Diabetes Ther 2016; 7:695-711.

10. Thayer S, Chow W, Korrer S, Aguilar R. Real-world evaluation of glycemic control among patients with type 2 diabetes mellitus treated with canagliflozin versus dipeptidyl peptidase-4 inhibitors. Curr Med Res Opin 2016; 32;1087-96.

11. Fioretto P, Giaccari A, Sesti G. Efficacy and safety of dapagliflozin, a sodium glucose cotransporter 2 (SGLT2) inhibitor, in diabetes mellitus. Cardiovasc Diabetol 2015; 14:142.

12. Zinman B, Wanner C, Lachin JM, et al; EMPA-REG OUTCOME Investigators. Empagliflozin, cardiovascular outcomes, and mortality in type 2 diabetes. $\mathrm{N}$ Engl J Med 2015; 373:2117-28.

13. Scheen AJ. Pharmacodynamics, efficacy and safety of sodium-glucose cotransporter type 2 (SGLT2) inhibitors for the treatment of type 2 diabetes mellitus. Drugs 2015; 75:33-59.

14. Thong KY, Jose B, Sukumar N, et al; ABCD Nationwide Exenatide Audit Contributors. Safety, efficacy and tolerability of exenatide in combination with insulin in the Association of British Clinical Diabetologists nationwide exenatide audit*. Diabetes Obes Metab 2011; 13:703-10.

15. Ji L, Ma J, Li H, et al. Dapagliflozin as monotherapy in drug-naive Asian patients with type 2 diabetes mellitus: a randomized, blinded, prospective phase III study. Clin Ther 2016; 36:84-100.e9.

16. Sherifali D, Nerenberg K, Pullenayegum E, Cheng JE, Gerstein HC. The effect of oral antidiabetic agents on A1C levels: a systematic review and meta-analysis. Diabetes Care 2010; 33:1859-64.

17. Liang Y, Arakawa K, Ueta K, et al. Effect of canagliflozin on renal threshold for glucose, glycemia, and body weight in normal and diabetic animal models. PLoS One 2012; 7:e30555. 